\title{
EcoBank: A Flexible Data Platform for Sharing Ecological Data
}

\author{
Hyun Woo Kim ${ }^{1}$, Sungsoo Yoon ${ }^{1}$, Mokyoung Kim ${ }^{1}$, Man-seok Shin ${ }^{2}$, Heenam Yoon ${ }^{1}$, and \\ Kidong $\mathrm{Kim}^{1}$ \\ ${ }^{1}$ National Institute of Ecology \\ ${ }^{2}$ Affiliation not available
}

November 9, 2020

\begin{abstract}
Environmental crisis challenges human race harder than ever before. Ecologists have produced a massive amount of data to cope with the crisis. Accordingly, so many ecological data platforms have been developed worldwide to manage and analyze these datasets. However, in Korea, ecological datasets produced by different research institutes for different purposes have not been integrated or serviced due to the lack of a well-designed information infrastructure. To address this obstacle, we present EcoBank (www.nie-ecobank.kr), an open, web-based ecological data platform designed to play an important role in ecological informatics in Korea. The architecture of EcoBank comprises core technologies of WebGIS, Application Programming Interface (API), responsive web, and open-source software (OSS). EcoBank has three conceptual modules: data management, analysis, and service. Diverse stakeholders of EcoBank can be classified into three groups: researchers, policymakers, and public users. EcoBank aims to expand its horizons through mutual communication between these stakeholders. As a part of the progress, EcoBank began to broaden its network by linking to other data platforms and repositories over the globe to find possible solutions to ecological issues in Korea.
\end{abstract}

\section{EcoBank: A Flexible Data Platform for Sharing Ecological Data}

Running Head: The first ecological data platform in Korea

Hyun Woo Kim*, Sungsoo Yoon**, Mokyoung Kim**, Manseok Shin**, Heenam Yoon***, Kidong Kim****

* Research Specialist, EcoBank Team, National Institute of Ecology, South Korea

** Researcher, EcoBank Team, National Institute of Ecology, South Korea

*** Senior Researcher, EcoBank Team, National Institute of Ecology, South Korea

**** Chief Researcher, Division of Ecological Information, National Institute of Ecology, South Korea

Corresponding author: Kidong Kim, email:kidong@nie.re.kr

(Submitting author: Hyun Woo Kim, email:khw4eco@nie.re.kr)

\section{Abstract}

Environmental crisis challenges human race harder than ever before. Ecologists have produced a massive amount of data to cope with the crisis. Accordingly, so many ecological data platforms have been developed worldwide to manage and analyze these datasets. However, in Korea, ecological datasets produced by different research institutes for different purposes have not been integrated or serviced due to the lack of a well-designed information infrastructure. To address this obstacle, we present EcoBank (www.nieecobank.kr), an open, web-based ecological data platform designed to play an important role in ecological 
informatics in Korea. The architecture of EcoBank comprises core technologies of WebGIS, Application Programming Interface (API), responsive web, and open-source software (OSS). EcoBank has three conceptual modules: data management, analysis, and service. Diverse stakeholders of EcoBank can be classified into three groups: researchers, policymakers, and public users. EcoBank aims to expand its horizons through mutual communication between these stakeholders. As a part of the progress, EcoBank began to broaden its network by linking to other data platforms and repositories over the globe to find possible solutions to ecological issues in Korea.

KEYWORDS: EcoBank, ecological data platform, responsive web, WebGIS, diverse stakeholders

\section{Introduction}

The ultimate goal of ecological studies is to understand and quantify ecological associations in different spatiotemporal scales (Kissling et al., 2012; Stephens et al., 2019). However, issues in ecology are becoming complicated due to various and deleterious anthropogenic effects such as land cover change, global warming, and environmental pollution (Araújo \& Rahbek, 2006; Borges et al., 2020; de Chazal \& Rounsevell, 2009; Parmar, Rawtani, \& Agrawal, 2016). Thus, it is important to secure accurate and up-to-date knowledge of the ecosystem.

Ecologists have collected data through various methods in different fields of ecology to address complex environmental problems (H. W. Kim, Hwang, Mu, Lee, \& Choi, 2012; Lin, Feng, Liang, \& Ren, 2013; Nadkarni, 1981; Y. M. Park, 1990; Thuiller, 2004). The world of big data has arrived in ecology and other branches of the environmental sciences (Farley, Dawson, Goring, \& Williams, 2018) with increased quantity, speed, and variety of data streams for ecological information (Hampton et al., 2013; Xu, Zhang, Gao, Yang, \& Shi, 2018). In the field of ecological informatics, various ecological data platforms have been developed worldwide with the aim to collect and manage ecological information efficiently.

Although a few web-based platforms have been designed for biodiversity conservation plans and biological resource management in Korea, significant challenges in addressing ecological issues remain (Table 1, Appendix 1). As there is no integrated platform for comprehensive utilization and one-point access to ecological data, these data are scattered throughout individual institutions, producing a particular type of data without consistent format or retrieval capability (Youn, 2014). In Korea, existing platforms do not use standardized ecological metadata for sharing or organizing ecological information. Additionally, many platforms have difficulties in providing user-oriented data due to the lack of open space and systems for quality control (i.e., BES-NET) (Ruckelshaus et al., 2020).

New challenges for the management of ecological data require improved interoperability, integration, and sharing (Frehner \& Brändli, 2006). An integrative and comprehensive data platform is strongly recommended in Korea. To address this need, we present EcoBank in three sections (background, architecture, and implementation) to show its extendible framework for exploration, modeling, and analysis of distributed ecological data in a Web-based environment. In the background section, EcoBank's developmental phases and core technologies are described. In the architecture section, the conceptual design of EcoBank is described and functions related to exchanging, sharing, and analyzing spatial data through the internet are discussed. The following implementation section describes functions and services mainly for target stakeholders. The paper ends with main conclusions and an outlook for future directions.

\section{Background - development process and core technologies}

\section{Development process}

We developed EcoBank through three developmental process phases (Appendix. 2). In the first phase (20142018), EcoBank development began with information strategy planning which addressed long-term management of its data, information, and knowledge. Target analysis was also conducted with various internal and external expert groups to investigate opportunities of EcoBank as an international platform in the global market. Main system, including a web geographic information system (WebGIS) and a policy support tool, was developed. Integration of existing domestic databases into EcoBank began in this phase. In the second 
phase (2019-2020), EcoBank was officially launched and widely accepted by target stakeholders. EcoBank provides open API services for application to any type of data platform. EcoBank has broadened its connections to the East Asian region with various cooperative projects. Additionally, EcoBank developers have suggested an ecological metadata standard based on Data Catalog Vocabulary (DCAT). In the third phase $\left(2021^{\sim}\right)$, EcoBank is expected to gain more advanced functions and analysis tools in communication with its stakeholders. International ecological data standards can be derived from its experience and expertise in ecological data management.

\section{Core technologies of EcoBank}

In this section, core technologies including WebGIS, Open Source Software (OSS), open API, and responsive web to operate EcoBank properly and efficiently are briefly explained. In the next section, how these technologies are integrated into EcoBank will be discussed.

Research efforts of geographic information science community have been made targeting an integration of ecological data (Frehner \& Brändli, 2006). Considering the massive increase of internet use, traditional GIS paradigm on spatial data handling from a single database has gradually shifted toward a distributed GIS paradigm through physically distributed database systems or geospatial services (Preston, Clayton, \& Wells, 2003). Technology of web services and open standards has provided the basis for distributed geoprocessing or distributed GIS. A step toward distributed GIS is the establishment of so-called geoportals that offer gateways to discover and access geographic web services (Maguire \& Longley, 2005). However, an examination of several existing geoportals has shown that the available functionality is restricted to searching, mapping, publishing, and limited querying of distributed geodata (Tait, 2005). A missing feature is the availability of comprehensive analysis tools. WebGIS, also known as Web-based GIS or Internet GIS (Peng \& Tsou, 2003), provides additional means for spatial data analysis as an alternative.

OSS is a type of computer software in which the source code is released under a license in which the copyright holder grants users the rights to study, change, and distribute the software to anyone for any purpose (Lakhani \& Von Hippel, 2004; Steiniger \& Hay, 2009). Almost all software packages used in EcoBank are based on open-source.

API may be defined as "an interface designed to control the functionality provided by the operating system or programming language for use by applications" (Chen, Annadata, \& Chan, 2009). It is an intermediary medium that helps our services use features and programs provided by individual developers, businesses, and organizations. If the user interface (UI) connects users and objects (hardware or software) to be handled by users, the API connects the program to another program. API is used in almost all ecological data platforms worldwide (Frehner \& Brändli, 2006; W. Michener et al., 2011; Stephens et al., 2019), and also used in EcoBank.

Optimizing any web-based information for various devices is no longer an option but a necessity. Due to increasingly diverse smart devices, it is impossible to provide an appropriate service to customers with a desktop-version website alone. Responsive web design is a method to effectively enable the use by various devices (Gardner, 2011). EcoBank takes advantage of the Responsive web design. Layouts are instantly transformed to the best design for many different web circumstances, such as mobile phones, tablets, and desktops. It not only provides adjustable sizes for webpages, but also optimizes components for their own operational principles.

\section{Integration of core technologies into EcoBank}

All core technologies were successfully integrated into EcoBank. WebGIS (Fig. 1) allows visualization of all spatial data (raster and vector) from three input data sources: NIE, other environmental institutes in Korea, and international platforms and repositories. WebGIS functions in EcoBank are composed of Geoserver for mapping service, OpenLayers for implementing map service, PostGIS for spatial operation and spatial quarry, and PostgreSQL for managing and storing spatial data. The WebGIS solution in EcoBank first collects various 
ecological information with diverse file formats such as shape (SHP), coma separated values (CSV), and text only (TXT), and loads them into PostgreSQL using PostGIS after diagnosis, transformation, and processing to meet ecological spatial data standard. These loaded spatial data are registered and issued in GeoServer, and provided as a map service of the Ecobank using OpenLayers technology. With this distributed GIS, EcoBank intends to have more user-centered functions that will not only present processed results, but also provide a chance to overlap data in EcoBank for its own purpose.

Almost all software packages applied in EcoBank (Fig. 2) are based on the open source architecture. For example, Red Hat Enterprise Linux is mounted on a physical web server, a web application server, and a linked server. The database server uses PostGIS, PostgreSQL, and Selenium a suite of tools for automating web browsers. KoNLPy, a Phython package for processing information in Korean language, is also applied in EcoBank for web crawling. Other software packages for ETL, Web server and GIS server, and Web Client are also OSS packages. EcoBank utilizes generalized and OSS packages to eliminate its dependence on software packages of specific companies and observes open standards.

Open API is also efficiently used in connecting and sharing data with other platforms and repositories worldwide. EcoBank provides various ecological spatial information in the form of Web Map Service (WMS) and Web Feature Service (WFS). The WMS service allows one to use map images with diverse formats such as Portable Network Graphics (PNG), Joint Photographic Experts Group (JPG), and Graphics Interchange Format (GIF) generated from geographic data. The WFS service makes it possible to utilize geographic feature data with vector figures and attributes. To use the OpenAPI of the EcoBank system, an authentication key is needed for each layer to be used. It can be used immediately after obtaining approval from the administrator. In addition, EcoBank supports the use of OpenAPI more easily by providing sample codes of programming languages such as Java, JavaScript, Hypertext Preprocessor (PHP), and Python for the use of ecological spatial information. Through the API service, EcoBank has already begun linking its data to the biggest data repository in Korea's public sector (Public Data Portal, www.data.go.kr) without any extra processes. Data from EcoBank have been directly transferred and embodied in a different framework. They continue to work like its own data. API is a powerful tool for activating EcoBank's vision of facilitating data use in any other platform in the world. Anyone interested in developing ecological applications using features and datasets in EcoBank can freely utilize API services of EcoBank.

Responsive web design is also applied to EcoBank. The design concept in the EcoBank website is grouping visual images, infographics, and corresponding contents that can be perceived intuitively. The layout of EcoBank's main page has a vertical grid structure. A content group is deployed according to access frequency of the user. The EcoBank layout was optimized for 1600 px. In the meanwhile, the responsive web design leads us to create a UI for accessibility from all devices by focusing on aesthetics and convenience as much as possible.

Based on core technologies briefly explained above, EcoBank eliminates dependencies and complies with open standards utilizing open-source based, generalized, and open technologies. It guarantees interoperability by providing standards that can be linked to commercial solutions. EcoBank aims for national standardization. It can be replaced by modularization of each service, enabling flexible responses to changes and supporting convenient and diverse environments, such as Eclipse-based modeling, editing, compilation, and debugging environments.

\section{Conceptual Architecture of EcoBank}

In this section, the conceptual architecture of EcoBank is presented (Fig. 3). One of primary goals of EcoBank is to function as the main access point for data and data resources from the National Institute of Ecology (NIE), domestic, and international databases (Table 2). NIE itself already has a huge amount of heterogeneous datasets such as the National Ecosystem Survey (NES), the Ecosystem and Nature Map (ENM), the Intensive Ecological Survey (IES), the current status of invasive and ecosystem-disturbing species, and results from the National Long-Term Ecological Research projects. The NES, the largest survey project in Korea, has been conducted by the Korean Ministry of Environment for the mainland of South Korea since 
1986. ENMs are graded ( $1-3$ grades and separate management areas) for the natural environment on the basis of ecological and landscape values for mountains, rivers, inland wetlands, lakes, farmland, and cities. The Korean Ministry of Environment commissioned the NIE to conduct IES to scrutinize biodiversity in national protected areas such as Ecosystem \& Landscape Protection Areas and specific areas including some islands and coastal sand dunes. These datasets are sent to EcoBank after a rigorous quality control process.

EcoBank is exchanging ecological data with other environmental institutes in Korea such as the National Institute of Biological Research (NIBR) to support the implementation of national policies on biological resources. EcoBank is also linked to a public data portal (www.data.go.kr) which integrates Korean government's open data by providing access points on a web-based platform. Thus, the link with PDP can give more opportunities for people to access ecological data collected by NIE. EcoBank is using API services provided by Vworld (www.vworld.kr), an open platform service on spatial information operated by Spatial Information Industry Promotion Institute in Korea. Regarding areas in Korea, EcoBank can promote GIS services due to higher resolution and more up-to-date spatial information of Vworld maps compared to Google Maps.

EcoBank began to link with international platforms and repositories to boost the participation of international stakeholders in finding possible solutions of ecological issues in Korea. Ecological data from Kasetsart University in Thailand and from Nong Lam University in Vietnam were stored in EcoBank recently, and other repositories in Asian countries and global data platforms such as GBIF will also be connected in the near future. Data collected from these three routes will be linked to the main server of EcoBank through WebGIS or open API. To handle this diverse and complex stream of incoming data, EcoBank has applied three separate modules (Fig. 3). Compositions and purpose of each module are described in the following sections.

\section{Data management module}

Incoming datasets from the aforementioned three different routes arrive first in a data management module. These datasets need to be organized with standardized metadata for quality assurance and quality control. Due to the absence of any ecological metadata standards in Korea to manage domestic and foreign ecological data in an integrative way, we proposed a metadata schema for managing and sharing ecological data with Korean Telecommunications Technology Association (TTA). This schema will be a useful standard for efficient data management and interoperability both inside and outside ecological fields. Digital object identifiers (DOI) will be firstly assigned to data produced from NIE's research projects. DOI enables permanent access, precise identification, and reliable citation of data through EcoBank. DOI will be then assigned to ecological data published by GEO DATA (geodata.kr), the first data journal on earth science, ecology, ocean, aerospace, and polar research in Korea.

Datasets managed by metadata and labeled by DOI in the management module may also be analyzed statistically. Point-based ecological datasets such as NES, invasive species, and living modified organisms will be linked with spatial data including ENM, digital elevation, and land cover. They can be used for statistical analysis.

\section{Analysis module}

Analysis module is implemented within EcoBank with visualized statistical results and Web GIS-based analysis tools. Datasets after passing through the quality control process in the management module are used for analyzing biodiversity and modeling species distribution in the Analysis module. Some data produced can be used to evaluate the ENM. Basic ecological analyses on biodiversity, species distribution, and population density can be performed with the collected information. In addition, researchers can conduct species distribution modeling (SDM) studies using EcoBank data with well-known SDM models such as MaxEnt, the Generalized Additive Model (GAM), and Gap Analysis Program (GAP). EcoBank enables a nationwide distribution of species occurrence points to be checked. It also provides data on the ecological status of each branch for each species. These biodiversity datasets can be used to predict species distribution and habitat suitability. 


\section{Service module}

EcoBank's various services for its users are categorized into data-sharing, public participation, and policy decision-making support. Open data sharing occurs between citizens through various data boards and Open API services. Furthermore, each open dataset that can be identified by DOI is permanently citable and trackable for users. EcoBank specifies multiple user groups to grant access to data containing sensitive information such as endangered species, natural reserves, and roadkill photos. These layered services meet needs and usage objectives of various stakeholders in a data platform $(\mathrm{Li}, \mathrm{Li}, \mathrm{Yu}$, \& Xie, 2010; W. K. Michener et al., 2012). To promote public participation, EcoBank has built discussion boards for ecological topics and uploaded species observation data. Currently, the NES research project actively supports ecological expertise to improve the quality of data collected by citizen scientists. Similarly, ecological experts can broaden their ideas or insights regarding ecological issues by discussing with citizens on EcoBank. Policymakers who have the same interests can plan environmental policies by exchanging ecological information through assigned EcoBank functions. Policy decision support services include opening data produced from environmental policies to the public and getting feedback from citizens.

\section{Target users/stakeholders}

A data platform may sound much superior if it has target users as many as possible as it needs to encompass diverse stakeholder communities. Thus, Michener et al. (2012) have proposed one primary stakeholder (scientists), five science research environments including academia, government, private industry, non-profit, and community, and over 20 end secondary stakeholders. However, DataONE is mainly focused on integrative biological and environmental research. Scientific research is only a small portion of the direction of EcoBank. If we follow similar approach of DataONE with a wide variety of stakeholders, it may be too complicated and digressive. After consulting with data platform experts, we have realized that it is necessary to start with certain targets to kick off a new data platform. Thus, we have reached to a decision to clarify target users into three groups: researchers, policymakers, and public users. It is possible to include more target users to meet needs of the market in the future.

\section{Researchers}

Professional researchers can find diverse data from various datasets in EcoBank produced by many different research projects. It contains NES data, Ecological Corridor data, Long-Term Ecological Research data, Bird Window Collisions Data, and Wetlands Data in Korea. One advantage of EcoBank is that all these datasets are integrated into one platform and presented with spatial information, enabling further studies in many broad dimensions. EcoBank also benefits researchers by offering analysis tools on the website. Researchers can explore the data and simultaneously analyze and generate results in a report. Except for data of endangered species, all data searchable in EcoBank are freely available to download and share.

Researchers may want to access data for a study species, learn about methods, run analyses, and share results with colleagues. With species occurrence data provided by EcoBank, researchers can carry out SDM studies, especially biodiversity changes influenced by climate changes, land-use changes, and environmental pollution. Data on ecological statuses can be downloaded and used for environmental impact assessment. Thus, it is possible to check national distribution of species occurrence points and download ecological status data for each species in EcoBank. This information may be used to predict potential species distribution and study habitat suitability.

\section{Policymakers}

Ecological research has to include the complex nature of disturbances and stability. Results of such multidimensional approaches can inform policymakers (Donohue et al., 2016). EcoBank also provides important ecological information to support decision-making in a user-friendly interface. EcoBank presents its data according to the administrative district of Korea so that policymakers can access important ecological information for the area of interest. Furthermore, the toolbar on the EcoBank website provides easily accessible and extra layers of information to instantly gain scientific support during a policy-making process. 
Ecological data with high credibility and accessibility are essential in this process. EcoBank provides observation density information on target species to facilitate the establishment of biodiversity conservation areas and the development of processes to help national environmental planning. EcoBank's biodiversity-related indices include Shannon-Wiener Biodiversity Index (E. C. Pielou, 1969), Dominance Index (McNaughton, 1967), Uniformity Index (E. Pielou, 1975), and Abundance Index (Margalef, 1958). For more information on biodiversity-related indices in EcoBank, see another article (Sung, Kwon, \& Kim, 2018).

\section{Public users}

Public users of EcoBank can be divided into three groups: students, business owners, and citizen scientists. EcoBank is applicable as an educational material. The curriculum for young students in Korea includes environmental education. However, few online materials have been developed for this subject. EcoBank allows free access to all ecological data that can be utilized for any purpose. For example, teachers can develop an educational program using ecological data to investigate the environmental impact of human behaviors. Conversely, students can generate creative ideas from data simulations in EcoBank.

For business owners, ecological issues and interests have led to the formation of a commodity market in the ecological information field. The related industry such as ecotourism has been largely expanded. However, without an efficient sharing and management of ecological data and information, time and resources are unavoidably wasted in a constant manner. EcoBank can benefit business owners by saving time and money to access data that they need through ENM.

EcoBank can promote citizen science within its function "Community." Citizen science may be defined as a scientific process conducted by many citizens in cooperation with or under the guidance of professional scientists or scientific institutions (Oxford English Dictionary, 2014). EcoBank provides a stable repository for citizen scientists. It also assures that citizen scientists can upload their findings under the guidance of professional researchers at the NIE to maintain data quality standards.

Citizen scientists have recorded their observations of the natural world, including species distribution, phenology, and climate data for centuries (Miller-Rushing, Primack, \& Bonney, 2012). As ecological research has grown into a relatively recent area of expertise, the contribution of citizen scientists to ecology is obviously apparent in history but easily overlooked. Researchers are currently reviewing numerous datasets collected by non-experts to identify long-term changes in the ecosystem. However, citizen science in Korea has remained very limited with a relatively low level of civic participation compared to that in European countries and the U.S. (J. H. Park, 2018). EcoBank may play an integral role in developing citizen science in Korea. Citizen scientists in Korea participating in any ecological data collection project, for instance, may use EcoBank data as a reference before conducting field studies.

\section{Possible applications using EcoBank for stakeholders}

We presented possible applications for each stakeholder. However, those examples are not strictly limited to individual categories, but integrative to each other. For example, researchers and policymakers have raised the need to study the distribution of herptile (including both reptile and amphibian) species with multi-scale (Fig. 4) regarding ecosystem conservation. Studies have highlighted that the distribution of herptile species can be the key to develop a universal ecological integrity (EI) for evaluating the ecological health of aquatic and terrestrial ecosystems (Ali, Javid, Hussain, \& Bukhari, 2018). In addition, research on detrimental effects of American bullfrog (Kang, Koo, \& Sung, 2019) has addressed the spatial distribution of this frog species (Fig. 5). EcoBank is an excellent information source for this purpose as it provides spatial information of diverse organisms including herptiles with multi-scale from nationwide to smaller administrative units in Korea (Fig. 4).

With ecotourism in the spotlight recently, Jeju Island where the tourism industry accounts for more than a quarter of its total economic production might highly depend on extraordinary ecosystems comprising numerous rare, protected flora, and fauna (E.-M. Kim, Choi, \& Kang, 2013). ENM gives land use status and improves our general understanding of natural environments in Jeju island (Fig. 6(a)). By selecting areas of 
high ecological value, it is possible to maintain and increase ecosystem services and obtain economic benefits from natural asset use. Therefore, ecological grade information in ENM can be utilized by policymakers to prepare a land-use plan that regulates excessive developments. Public users will be able to predict and plan for land-use projects where development can be restricted in a similar way (Ahn et al., 2015).

The use of airspace has caused spatial conflict between birds and people. Bird collisions resulting from artificial structures are prime examples of this issue. Thus, researchers need scientific data to understand the status and annual mortality estimates of bird collision which has been poorly understood in Korea (Seo, 2020). Records of bird collisions can be retrieved from newspapers, verbal communication, online forums, and blog posts. They are frequently collected by citizens. These data can be analyzed to publish significant results regarding risk factors and bird collisions patterns in scientific journals (Basilio, Moreno, \& Piratelli, 2020; Low, Yong, Tan, Owyong, \& Chia, 2017; Rebolo-Ifrán, di Virgilio, \& Lambertucci, 2019). Although the quality of data produced from citizen scientist has long been a concern due to the lack of quality control process (Kosmala, Wiggins, Swanson, \& Simmons, 2016), Bird Window Collision data have been successfully collected within a research project through EcoBank (Fig. 6(b)). With increasing need for big data, such collaborative projects are expected to deal with ongoing ecological problems.

\section{Conclusions and future directions}

In this article, we present EcoBank, an open-accessible data platform (http://nie-ecobank.kr) that is sufficiently flexible, easy to operate, and user friendly to facilitate different types of needs. It allows the exploration and incorporation of ecological data and its subsequent integration into predictive models for

both potential ecological niches and geographic distribution. It also provides online analyses that might be applied to develop various environmental policies or may guide researchers to identify relationships between different biota. EcoBank is constantly updating latest ecological research data and improving user convenience functions through mutual communications with stakeholders. Through its ongoing development as an integrated international ecological information system, EcoBank will deliver accumulated technology to countries without any ecological information system. We will work closely with a cadre of international data management groups and continuously establish international links to ecological information. We hope that EcoBank will become an avenue for international stakeholders to obtain ecological information in Korea.

\section{Acknowledgments}

This study was conducted in the National Institute of Ecology with financial support from the Ministry of the Environment (NIE-Strategic Research-2020-01) and the ICT promotion fund of Korean Ministry of Science and ICT. We want to thank Jae Yu Yang and Sung Ho Suh from Wavus Co., Korea for their technical support and invaluable advices during the development of this work.

\section{Data Accessibility}

All spatial datasets including species occurrence data and background maps used in the figures in the main text and the supplementary information session of this manuscript are publicly available on the EcoBank webpage at the following URL:

https://www.nie-ecobank.kr/cmmn/Index.do?

\section{References}

Ahn K, Shin Y, Kim J, Lee Y, Lim J, Ha J, Kwon H, Suh J, Kim M. 2015. A Review on the Public Appeals of the Ecosystem and Nature Map. Journal of Environmental Impact Assessement. 24(1):99-109. Korean.

Ali W, Javid A, Hussain A, Bukhari SM. 2018. Diversity and habitat preferences of amphibians and reptiles in Pakistan: a review. Journal of Asia-Pacific Biodiversity. 11(2):173-187.

Araujo MB, Rahbek C. 2006. How Does Climate Change Affect Biodiversity? Science.313:1396-1397.

Basilio LG, Moreno DJ, Piratelli AJ. 2020. Main causes of bird-window collisions: a review. An Acad Bras Cienc.92(1). 
Chen M, Annadata AK, Chan L. 2009. Adaptive communication application programming interface. In: Google Patents.

de Chazal J, Rounsevell MDA. 2009. Land-use and climate change within assessments of biodiversity change: A review. Global Environmental Change. 19(2):306-315.

Donohue I, Hillebrand H, Montoya JM, Petchey OL, Pimm SL, Fowler MS, Healy K, Jackson AL, Lurgi M, McClean D, et al. 2016. Navigating the complexity of ecological stability. Ecology Letters 19(9):1172-1185.

Farley SS, Dawson A, Goring SJ, Williams JW. 2018. Situating Ecology as a Big-Data Science: Current Advances, Challenges, and Solutions. BioScience. 68(8):563-576.

Frehner M, Brandli M. 2006. Virtual database: Spatial analysis in a Web-based data management system for distributed ecological data. Environmental Modelling and Software. 21(11):1544-1554.

Gardner BS. 2011. Responsive web design: Enriching the user experience. Sigma Journal: Inside the Digital Ecosystem.11(1):13-19.

Hampton SE, Strasser CA, Tewksbury JJ, Gram WK, Budden AE, Batcheller AL, Duke CS, Porter JH. 2013. Big data and the future of ecology. Frontiers in Ecology and Environment. 11(3):156-162.

Kang H-J, Koo KS, Sung H-C. 2019. Current distribution of American bullfrog Rana catesbeiana Shaw, 1802 in the Republic of Korea. BioInvasions Records. 8(4):942-946.

Kim E-M, Choi C-Y, Kang C-W. 2013. Causes of injury and mortality of Fairy Pitta Pitta nympha on Jeju Island, Republic of Korea. Forktail. 29:145-148.

Kim HW, Hwang K, Mu Q, Lee SO, Choi M. 2012. Validation of MODIS 16 global terrestrial evapotranspiration products in various climates and land cover types in Asia. KSCE Journal of Civil Engineering. 16:229-238.

Kissling WD, Dormann CF, Groeneveld J, Hickler T, Kuhn I, McInerny GJ, Montoya JM, Romermann C, Schiffers K, Schurr FM, et al. 2012. Towards novel approaches to modelling biotic interactions in multispecies assemblages at large spatial extents. Journal of Biogeography. 39:2163-2178.

Kosmala M, Wiggins A, Swanson A, Simmons B. 2016. Assessing data quality in citizen science. Frontiers in Ecology and Environment. 14:551-560.

Lakhani KR, Von Hippel E. 2004. How open source software works: "free" user-to-user assistance. In: Produktentwicklung mit virtuellen Communities. Springer. p. 303-339.

Li G, Li C, Yu W, Xie J. 2010. Security accessing model for web service based geo-spatial data sharing application. $3^{\text {rd }}$ Digital Earth Summit, ISDE, Nessebar, Bulgaria.

Low B, Yong D, Tan D, Owyong A, Chia A. 2017. Migratory bird collisions with man-made structures in South-East Asia: a case study from Singapore. BirdingASIA. 27:107-111.

Maguire DJ, Longley PA. 2005. The emergence of geoportals and their role in spatial data infrastructures. Computers, Environment, and Urban Systems. 29:3-14.

Margalef R. 1958. Information theory in ecology. International Journal of General Systems. 3:36-71.

McNaughton S. 1967. Relationships among functional properties of Californian grassland. Nature. 216:168169.

Michener W, Vieglais D, Vision T, Kunze J, Cruse P, Janee G. 2011. DataONE: Data Observation Network for Earth-Preserving data and enabling innovation in the biological and environmental sciences. D-Lib Magazine.17. doi:10.1045/january2011-contents 
Michener WK, Allard S, Budden A, Cook RB, Douglass K, Frame M, Kelling S, Koskela R, Tenopir C, Vieglais DA. 2012. Participatory design of DataONE - Enabling cyberinfrastructure for the biological and environmental sciences. Ecological Informatics. 11:5-15.

Miller-Rushing A, Primack R, Bonney R. 2012. The history of public participation in ecological research. Frontiers in Ecology and Environment. 10(6):285-290.

Nadkarni NM. 1981. Canopy Roots: Convergent Evolution in Rainforest Nutrient Cycles. Science. 214:10231024 .

Park JH. 2018. The Current state and tasks of citizen science in Korea. Journal of Science and Technology Study. 18(2):7-41. Korean.

Park YM. 1990. Effects of Drought on Two Grass Species with Different Distribution Around Coastal Sand-Dunes. Functional Ecology. 4(6):735-741.

Parmar TK, Rawtani D, Agrawal YK. 2016. Bioindicators: the natural indicator of environmental pollution. Frontiers in Life Science. 9(2):110-118.

Peng Z-R, Tsou M-H. 2003. Internet GIS: distributed geographic information services for the internet and wireless networks: John Wiley \& Sons. Inc.

Pielou E. 1975. Ecological diversity. John Wiley \& Sons. Inc.

Pielou EC. 1969. An introduction to mathematical ecology. Wiley Interscience.

Preston M, Clayton P, Wells G. 2003. Dynamic run-time application development using CORBA objects and XML in the field of distributed GIS. International Journal of Geographical Information Science. 17(4):321341.

Rebolo-Ifran N, di Virgilio A, Lambertucci SA. 2019. Drivers of bird-window collisions in southern South America: a two-scale assessment applying citizen science. Nature Scientific Reports. 9:1-10.

Ruckelshaus MH, Jackson ST, Mooney HA, Jacobs KL, Kassam K-AS, Arroyo MTK, Baldi A, Bartuska AM, Boyd J, Joppa LN, et al. 2020. The IPBES Global Assessment: Pathways to Action. Trends in Ecology and Evolution. 35(5):407-414.

Seo H. 2020. Bird collision with transparent structures in the Republic of Korea: current status and annual mortality estimates [Master's Thesis]. Seoul National University. Korean.

Steiniger S, Hay GJ. 2009. Free and open source geographic information tools for landscape ecology. Ecological Informatics. 4(4):183-195.

Stephens CR, Sierra-Alcocer R, Gonzalez-Salazar C, Barrios JM, Salazar Carrillo JC, Robredo Ezquivelzeta E, del Callejo Canal E. 2019. SPECIES: A platform for the exploration of ecological data. Ecology and Evolution. 9(4):1638-1653.

Sung S, Kwon YS, Kim K. 2018. Development and Applications of Ecological Data Portal Service (EcoBank) for Sharing Ecological Information of Korea. Korean Journal of Ecology and Environment. 51(3):212-220. Korean.

Tait MG. 2005. Implementing geoportals: applications of distributed GIS. Computers, Environment and Urban Systems. 29(1):33-47.

Thuiller W. 2004. Patterns and uncertainties of species' range shifts under climate change. Global Change Biology. 10(12):2020-2027.

Youn JH. 2014. The Establishment of BPR for National Spatial Data Infrastructure Quality Management System," Journal of Korean Society of Geospatial Information Science. 22(4):81-89. Korean.

\section{Hosted file}


Kim etal_20.1105_Figures.pdf available at https://authorea.com/users/374066/articles/491688ecobank-a-flexible-data-platform-for-sharing-ecological-data 